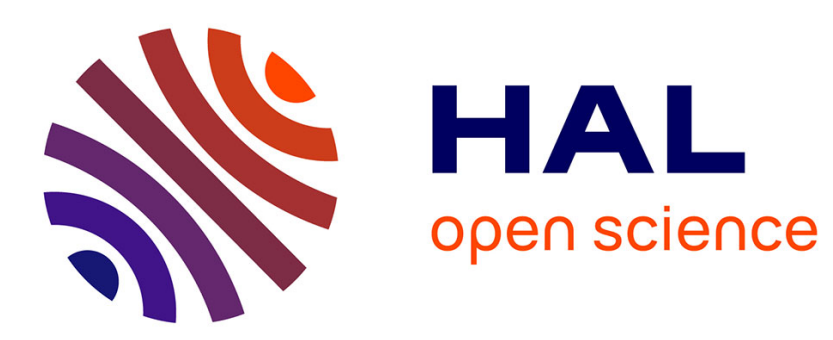

\title{
Une photographie du corps des professeurs des universités de sciences économiques en 2011
}

Thomas Jobert

\section{To cite this version:}

Thomas Jobert. Une photographie du corps des professeurs des universités de sciences économiques en 2011. 2012. hal-00728157

\section{HAL Id: hal-00728157 \\ https://hal.science/hal-00728157}

Preprint submitted on 5 Sep 2012

HAL is a multi-disciplinary open access archive for the deposit and dissemination of scientific research documents, whether they are published or not. The documents may come from teaching and research institutions in France or abroad, or from public or private research centers.
L'archive ouverte pluridisciplinaire HAL, est destinée au dépôt et à la diffusion de documents scientifiques de niveau recherche, publiés ou non, émanant des établissements d'enseignement et de recherche français ou étrangers, des laboratoires publics ou privés. 


\section{Une photographie du corps des professeurs des universités de sciences économiques en 2011.}

septembre 2012

\section{Thomas Jobert}

Université Nice Sophia Antipolis, 24 Avenue des Diables Bleus, 06357 Nice Cedex 4. e-mail: thomas.jobert@unice.fr

GREDEG, UMR de l'Université de Nice Sophia Antipolis et du CNRS (UMR 6227).

Résumé : La section CNU 05 (sciences économiques) est importante en terme d'effectifs, mais en perte de vitesse par rapport aux disciplines voisines (droit et gestion). Le concours d'agrégation externe permet de recruter des professeurs plus jeunes que dans les disciplines soumises à la règle commune, mais cet avantage est compensé par une évolution de carrière plus lente. En terme de répartition par corps d'appartenance ( $\mathrm{PR}$ ou $\mathrm{MdC})$, de sexe, et de région géographique d'exercice, nous sommes plutôt dans la moyenne, mais avec un peu trop de professeurs hommes-parisiens. La construction du tableau de classement au 31 décembre 2011, met en lumière à la fois le très faible taux de femmes dans la classe exceptionnelle, et une sous représentation des collègues nés durant la décennie 1951-1960 (avec des répercussions à venir au moment de leur départ à la retraite). L'analyse conjointe de la répartition par ancienneté dans chaque grade et des candidatures à la promotion entre 2009 et 2011, montre qu'un certain nombre de collègues en seconde ou première classe ont abandonné toute idée d'avancement . 


\section{Introduction.}

L'université française est en pleine mutation. Au niveau des établissements, la loi du 10 août 2007 relative aux libertés et responsabilités des universités, dite loi LRU, est en train de dessiner un nouveau paysage universitaire. Pour les enseignants chercheurs, la modification du décret du 6 juin 1984 (fixant les dispositions statutaires communes applicables aux enseignants-chercheurs et portant statut particulier du corps des professeurs des universités et du corps des maîtres de conférences) par le décret du 23 avril 2009 apporte de profonds changements dans leurs missions et obligations de services. Un plan en faveur des carrières dans l'enseignement supérieur et la recherche a pris effet en 2009 : la durée des échelons des professeurs des universités a été modifiée afin de la rendre strictement identique à celle des directeurs de recherche ${ }^{1}$. Le déroulement de carrière a été accéléré : le taux de promotion ${ }^{2}$ des professeurs à la première classe est progressivement passé de $10 \%$ en 2008 à $15 \%$ en 2011 et le taux de promotion des professeurs à la classe exceptionnelle de $8 \%$ en 2008 à $15 \%$ en 2011.

Ces réformes ne se font pas sans heurts suscitent de nombreuses controverses. Le monde des économistes fait aussi face à des débats plus spécifiques. Ainsi de nombreuses pétitions circulent au sujet du mode de recrutement professeurs par les concours d'agrégation, ou encore sur l'opportunité de créer une nouvelle section CNU intitulée économie et société.

L'objectif de ce travail est d'apporter des éléments d'information essentiels à ces débats en proposant une photographie aussi précise que possible sur le corps des professeurs des universités (PR) de la section 05 du CNU (Sciences Economiques). Dans une première partie, à partir de statistiques et de rapports du Ministère de l'Enseignement Supérieur et de la Recherche (MESR), nous esquissons l'arrière plan de cette photographie en analysant les évolutions des effectifs depuis quinze ans et en recherchant les caractéristiques du corps des PR de sciences économiques par rapport à celles des autres disciplines. Dans une seconde partie, grâce à la reconstitution du tableau de classement au 31 décembre 2011, nous proposons une analyse des trois grades du corps de PR de la section 5.

\section{L'arrière plan.}

Il s'agit de restituer la place des professeurs des universités dans l'évolution des effectifs des personnels enseignants du supérieur depuis une quinzaine d'années, puis de mettre en évidence certaines spécificités de la section CNU 05. Pour cela nous utilisons des données du

\footnotetext{
${ }^{1}$ L'ancienneté requise pour passer du 5e au 6e échelon de la 2e classe est de 3 ans et 6 mois au lieu de 5 ans. Le temps passé aux 1er et 2e échelons de la 1ère classe est de 3 ans au lieu de 4 ans et 4 mois.

${ }^{2}$ Le taux de promotion est calculé par le rapport entre le nombre de promouvables et les possibilités de promotion.
} 
Ministère de l'Education Nationale (MEN) et du Ministère de l'Enseignement Supérieur et de la Recherche (MESR). Cependant les données ne sont pas toujours homogènes et cohérentes entre elles, et les trois problèmes principaux sont d'abord la délimitation du champ des disciplines (se concentrer sur celles correspondant aux sections du CNU ou inclure la santé), ensuite le statut des Enseignants Chercheurs (titulaire, stagiaire, associé), et enfin le moment où le stock des effectifs est mesuré (année civile ou année universitaire).

\section{I.1. Les évolutions des personnels enseignants du supérieur}

Les données sont issues de l'édition 2011 des "Repères et références statistiques de sur les enseignements, la formation et la recherche", disponible sur la page web du MEN: http://www.education.gouv.fr/cid57096/reperes-et-references-statistiques.html

Entre 1995 et 2011, les personnels de l'enseignement supérieur ${ }^{3}$ ont globalement augmenté de $41 \%$.

Graphique 1 : Évolution des enseignants du supérieur public de 1995 à 2011 (base 100 en 1995)

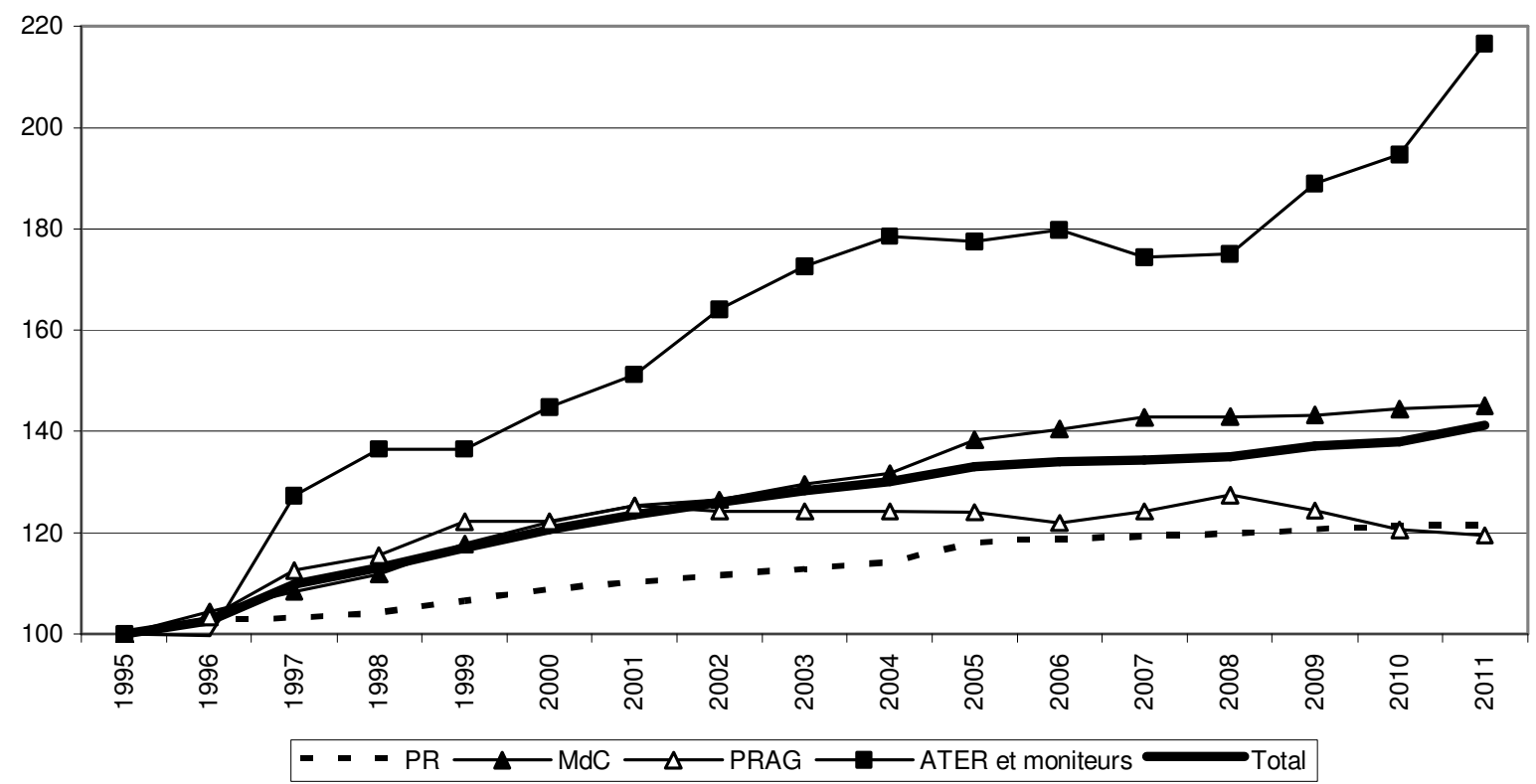

Source : Repères et références statistiques sur les enseignements, la formation et la recherche (2011).

Le graphique 1 montre une hausse assez régulière dans le temps des effectifs globaux, mais qui c'est faite au prix d'une nouvelle répartition au regard du statut spécifique des personnels. Les effectifs des PR croissent moins vite que la moyenne (+21.5\%) et stagnent depuis 2006 .

\footnotetext{
${ }^{3}$ Personnel enseignant dans les universités y compris les instituts universitaires de technologie (IUT), les écoles nationales supérieures d'ingénieurs (ENSI), les instituts universitaires de formation des maîtres (IUFM) rattachés aux universités, les écoles normales supérieures et les grands établissements.
} 
Avec une hausse de 45\%, l'évolution du nombre des maitres de conférences (MdC) est assez proche de celle de l'ensemble des effectifs. Les professeurs agrégés de l'enseignement du second degré affectés dans l'enseignement supérieur (PRAG) n'augmentent plus depuis 2001, et les effectifs ont même légèrement diminués. Le nombre des ATER (attachés temporaires d'enseignement et de recherche) et des moniteurs a explosé (+116\%).

En termes de parts, PR et MdC représentent environ deux tiers des effectifs. Alors que la part des MdC fluctue autours de $41 \%$, on assiste à une érosion de la place des PR dont la part diminue de $26 \%$ à $22 \%$. Les ATER et moniteurs sont maintenant plus nombreux que les PR et leur part progresse de $15 \%$ à 23\%. Les PRAG baissent légèrement de $16 \%$ à $13 \%$.

\section{I.2. Evolution des effectifs des enseignants-chercheurs (PR et MdC) de la section 5}

On se concentre maintenant uniquement sur les professeurs des universités et les maîtres de conférences répartis par section du CNU. Pour l'année universitaire 2009-2010 (le stock est mesuré au mois de mai 2010), la section 5 est la quatrième section du CNU en termes d'enseignants-chercheurs (1 827) derrière l'informatique (3 271), la mécanique (2 245) et les sciences de gestion (1 930).

\section{Graphique 2 : Évolution des effectifs d'enseignants-chercheurs titulaires ou stagiaires entre 1995 et 2010 (base 100 en 1995)}

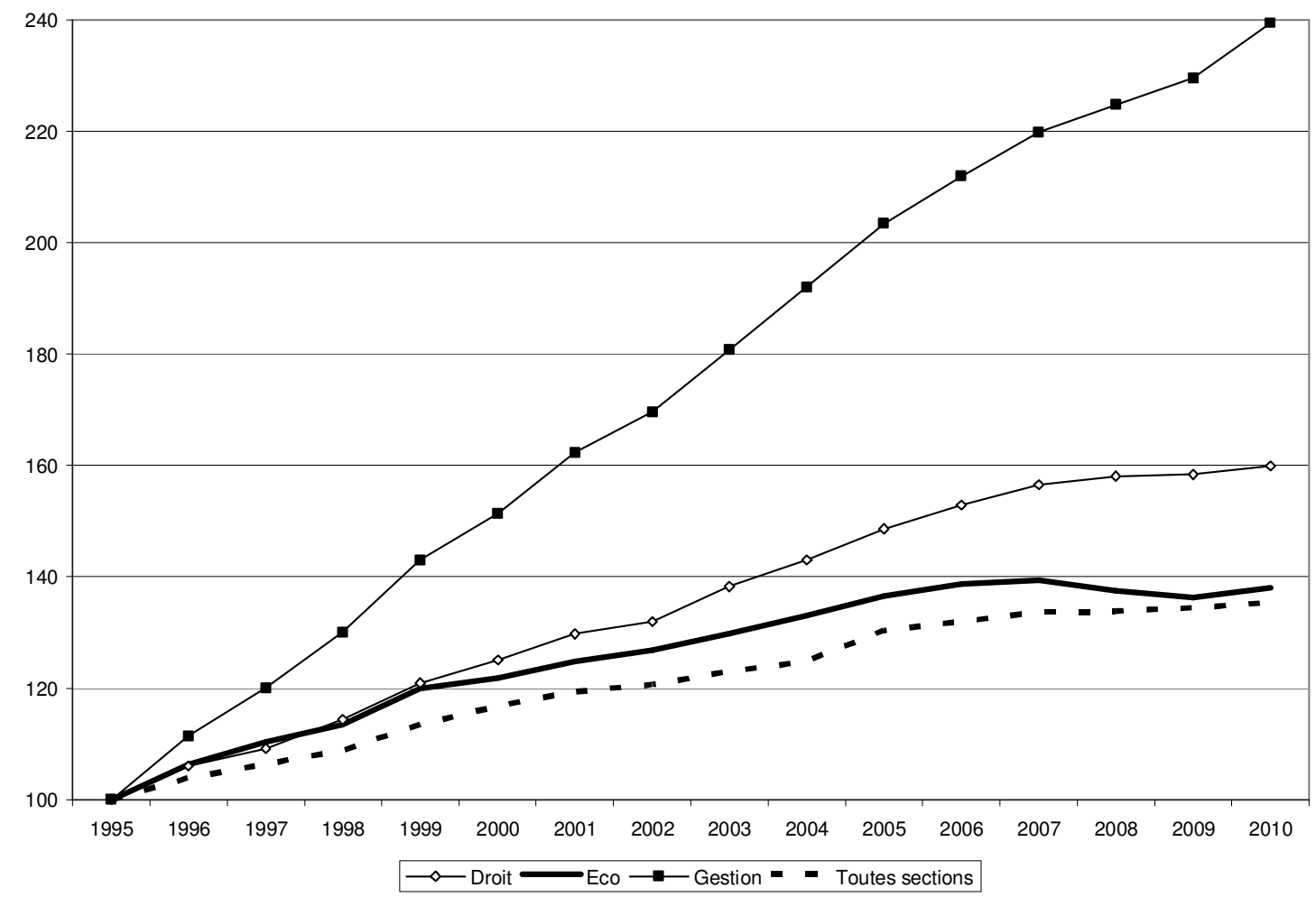

Source : Etudes et regards statistiques Démographie des enseignants de l'enseignement supérieur, 1996 à 2009. 
Le graphique 2 représente les évolutions des effectifs des enseignants-chercheurs (PR et MdC) de la section 5, de nos sections "cousines" (le droit et la gestion, qui recrutent aussi les PR par concours d'agrégation), et de l'ensemble des sections. En sciences économiques, les effectifs ont augmenté de $38.1 \%$, pour attendre 1827 enseignants-chercheurs. Comme les effectifs globaux n'ont augmenté que de $35.4 \%$, on peut penser que la discipline a été bien traitée. Cependant ce constat fait sur l'évolution globale cache trois faits marquants : d'abord les effectifs de la section 5 ont atteint un pic de 1844 EC en 2007 et décroissent depuis. Ensuite, relativement au droit et la gestion, nos effectifs croissent beaucoup moins vite : presque $+60 \%$ pour le droit (il s'agit des quatre sections du groupe 1) et $+140 \%$ pour la gestion. Enfin, alors qu'il y avait en 1995 environ deux tiers d'EC de plus en économie qu'en gestion, en 2009 le nombre d'EC en gestion (1850) a dépassé pour la première fois celui en économie (1802).

\section{I.3. Les spécificités de la section 5}

Une première spécificité des sciences économiques vient du mode de recrutement des PR (voir encadré 1). Alors que l'âge moyen d'entrée dans le corps des $\mathrm{MdC}$ des disciplines juridique, politique, économique et de gestion (les groupes 1 et 2 du $\mathrm{CNU}$ ) est quasiment le même que celui de l'ensemble des sections, grâce à ce mode spécifique de recrutement l'entrée dans le corps de $\mathrm{PR}^{4}$ est de 8 ans plus précoce (voir tableau 1). Ceci ne va pas être sans incidence sur l'avancement de grade dont les principales règles sont rappelées dans l'encadré 2.

Tableau 1 : Age moyen des lauréats de concours d'enseignants-chercheurs en 2008 par corps et par discipline

\begin{tabular}{|l|c|c|}
\hline & Maîtres de Conférences & Professeurs des universités \\
\hline Droit, Economie, Gestion & 32 ans 5 mois & 35 ans 9 mois \\
\hline Lettres et sciences humaines & 36 ans 5 mois & 47 ans 8 mois \\
\hline Sciences et Techniques & 30 ans 8 mois & 42 ans 4 mois \\
\hline Pharmacie & 31 ans 9 mois & 42 ans 5 mois \\
\hline Toutes disciplines confondues & 32 ans 9 mois & 43 ans 10 mois \\
\hline
\end{tabular}

Source : MESR / DGRH A1-1, ANTARES-ANTEE, juin 2008.

\section{Encadré 1 : Le recrutement des professeurs de sciences économiques}

Deux voies de recrutement sont offertes, les concours d'agrégation et les concours sur emplois.

1) les concours nationaux d'agrégation sur épreuves :

Un premier concours est destiné aux candidats titulaires d'un doctorat ou d'un diplôme équivalent (agrégation externe) ;

\footnotetext{
${ }^{4}$ Les PR recrutés en section 5 en 2010 (agrégation externe) ont un âge moyen de 38 ans et 4 mois et ceux de l'agrégation interne de 2011 un âge moyen de 44 ans et 1 mois. En 2011, toutes disciplines confondues, l'âge moyen auquel sont recrutés les nouveaux professeurs est aussi de 44 ans, 1 mois, et un MCF recruté PR a une ancienneté moyenne de 11 ans, 9 mois dans son corps.
} 
Un second concours est réservé aux maîtres de conférences âgés d'au moins 40 ans qui comptent 10 années de service dans l'enseignement supérieur et qui sont titulaires d'un doctorat ou d'un diplôme équivalent (agrégation interne).

2) les concours sur emplois :

Le troisième concours (le 46-3 ou la voie longue) est réservé aux maîtres de conférences titulaires de l'habilitation à diriger des recherches qui ont accompli 10 années de service dans un établissement d'enseignement supérieur. Le CNU formule a posteriori un avis sur les candidats retenus par l'établissement.

Pour le quatrième concours (le 46-4 ou la voie professionnelle) est ouvert aux candidats ayant 6 années d'activité professionnelle effective durant les 9 années précédentes (à l'exclusion des activités d'enseignant ou de chercheur), aux enseignants associés à temps plein, MdC membres de l'Institut universitaire de France, à des directeurs de recherche (sous certaines conditions). Le CNU qualifie les candidats ex ante.

\section{Encadré 2 : Les grades et l'avancement de grade}

Le corps des PR comporte une deuxième classe comprenant six échelons, une première classe comprenant trois échelons et une classe exceptionnelle comprenant deux échelons. L'avancement d'échelon dans la deuxième et la première classe a lieu à l'ancienneté. Les autres avancements ont lieu au choix et sont prononcés* pour moitié sur proposition de la section compétente du CNU (voie nationale) et pour moitié, sur proposition du conseil d'administration dans la limite des promotions offertes dans l'établissement, toutes disciplines confondues (voie locale).

Les règles de promouvabilité ont été fixées en 1987 et n'ont pas varié. L'avancement à la première classe a lieu au choix sans condition. L'avancement au premier échelon de la classe exceptionnelle ainsi que l'avancement au deuxième échelon de la classe exceptionnelle se fait au choix parmi les professeurs qui justifient d'au moins 18 mois d'ancienneté dans le grade inférieur. Les majorations d'ancienneté d'échelon et les bonifications d'ancienneté d'échelon dont les agents peuvent bénéficier viennent en déduction de cette durée.

Jusqu'en 2005, l'effectif de chacun des échelons de la classe exceptionnelle ne pouvait être supérieur à $10 \%$ de l'effectif total des professeurs. Le décret du 1 septembre 2005 modifie cette règle et introduit la notion de taux de promotion (le rapport entre le nombre des promus et celui des promouvables, c'est-à-dire des agents qui remplissent les conditions statutaires pour être promu au grade supérieur) fixé par un arrêté ministériel. Ainsi, le taux de promotion des professeurs à la première classe est passé de $10 \%$ en 2008 à $15 \%$ en 2011, et celui des professeurs à la classe exceptionnelle est passé de $8 \%$ en 2008 à $15 \%$ en 2011.

*Signalons qu'une voie spécifique d'avancement a été créée en 1989 pour des EC exerçants des fonctions particulières, autres que d'enseignement et de recherche (présidence d'un établissement, direction d'une UFR ou d'un institut, etc). Sur la période 2002-2009, 1.28\% des promotions à la première classe des professeurs ont été accordées par la voie spécifique, $2.46 \%$ des promotions au premier échelon de la classe exceptionnelle, et $3.59 \%$ des promotions au second échelon de la classe exceptionnelle.

Comme le montre le tableau 2, les promotions de grade dans les disciplines des groupes 1 et 2 sont plus longues à obtenir que dans les autres sections. Ainsi pour les promotions vers la première classe, au bout de 5 ans d'ancienneté presque un quart ont été données pour toutes les sections, alors que cela ne concerne que $16 \%$ des PR des groupes 1 et 2 . Malgré cela, l'âge moyen des promus à la première classe est plus jeune dans les disciplines avec recrutement par concours d'agrégation. Pour les promotions vers le premier échelon de la classe exceptionnelle, 25\% des lauréats ont moins de 7 ans d'ancienneté toutes disciplines confondues, et seulement $10 \%$ des groupes 1 et 2 . On remarque que ce 
temps supplémentaire d'attente ressert l'écart de l'âge moyen des promus entre les disciplines à un an. Enfin, pour le passage au deuxième échelon de la classe exceptionnelle, $66 \%$ des promotions sont distribués à des candidats ayant moins de 4 ans d'ancienneté, et l'âge moyen des promus tend à converger vers celui des autres sections.

Tableau 2 : Répartition des promotions par ancienneté dans le grade de 1993 à 2007.

\begin{tabular}{|l|c|c|c|c|c|c|}
\hline & \multicolumn{2}{|c|}{ PR2 vers PR1 } & \multicolumn{2}{c|}{ PR1 vers PREX1 } & \multicolumn{2}{c|}{ PREX1 vers PREX2 } \\
\hline $\begin{array}{l}\text { Ancienneté dans } \\
\text { le grade }\end{array}$ & $\begin{array}{c}\text { Toutes } \\
\text { sections }\end{array}$ & $\begin{array}{c}\text { Groupe } 1 \\
\text { et } 2\end{array}$ & $\begin{array}{c}\text { Toutes } \\
\text { sections }\end{array}$ & $\begin{array}{c}\text { Groupe } 1 \\
\text { et 2 }\end{array}$ & $\begin{array}{c}\text { Toutes } \\
\text { sections }\end{array}$ & $\begin{array}{c}\text { Groupe 1 } \\
\text { et 2 }\end{array}$ \\
\hline $\mathbf{1}$ ans & $0.4 \%$ & $0.29 \%$ & & & $2.12 \%$ & $1.78 \%$ \\
\hline $\mathbf{2}$ ans & $2.12 \%$ & $0.29 \%$ & $0.55 \%$ & & $16.35 \%$ & $12.76 \%$ \\
\hline $\mathbf{3}$ ans & $3.78 \%$ & $1.93 \%$ & $1.24 \%$ & $0.84 \%$ & $25.00 \%$ & $25.22 \%$ \\
\hline $\mathbf{4}$ ans & $6.55 \%$ & $4.92 \%$ & $2.96 \%$ & $0.42 \%$ & $21.88 \%$ & $23.15 \%$ \\
\hline $\mathbf{5}$ ans & $10.84 \%$ & $8.59 \%$ & $4.88 \%$ & $2.31 \%$ & $15.88 \%$ & $14.24 \%$ \\
\hline $\mathbf{6}$ ans & $13.82 \%$ & $13.03 \%$ & $6.57 \%$ & $3.15 \%$ & $9.41 \%$ & $8.61 \%$ \\
\hline $\mathbf{7}$ ans & $12.85 \%$ & $17.95 \%$ & $9.97 \%$ & $3.57 \%$ & $5.00 \%$ & $8.01 \%$ \\
\hline $\mathbf{8}$ ans & $12.43 \%$ & $15.35 \%$ & $11.21 \%$ & $5.88 \%$ & $1.65 \%$ & $1.7 \%$ \\
\hline 9ans & $9.51 \%$ & $13.03 \%$ & $10.97 \%$ & $6.72 \%$ & $0.94 \%$ & $1.19 \%$ \\
\hline $\mathbf{1 0}$ ans & $7.37 \%$ & $8.30 \%$ & $10.31 \%$ & $6.30 \%$ & $0.59 \%$ & $1.19 \%$ \\
\hline De 11 à 15 ans & $15.12 \%$ & $14.49 \%$ & $33.04 \%$ & $49.8 \%$ & $1.18 \%$ & $2.08 \%$ \\
\hline De 16 à 20 ans & $2.75 \%$ & $1.64 \%$ & $8.04 \%$ & $19.86 \%$ & $0 \%$ & $0 \%$ \\
\hline Plus de 20 ans & $1.48 \%$ & $0.20 \%$ & $0.28 \%$ & $0.63 \%$ & $0 \%$ & $0 \%$ \\
\hline $\begin{array}{l}\text { Age moyen des } \\
\text { promus }\end{array}$ & $\begin{array}{c}51 \text { ans } 2 \\
\text { mois }\end{array}$ & $\begin{array}{c}48 \text { ans } 2 \\
\text { mois }\end{array}$ & $\begin{array}{c}56 \text { ans } 8 \\
\text { mois }\end{array}$ & $\begin{array}{c}55 \text { ans } 10 \\
\text { mois }\end{array}$ & $\begin{array}{c}58 \text { ans } 3 \\
\text { mois }\end{array}$ & $\begin{array}{c}57 \text { ans } 5 \\
\text { mois }\end{array}$ \\
\hline
\end{tabular}

Source : "Etude de la promotion dans la carrière des enseignants-chercheurs 1993-2007", MESR juin 2009.

Le tableau 3 fournit plusieurs statistiques permettant de caractériser la section 5 relativement à l'ensemble des autres sections selon des trois grands critères : le corps d'appartenance (PR ou $\mathrm{MdC}$ ), le sexe, et région géographique d'exercice (Ile de France ou autres régions). Avec une part des PR de $29.5 \%$ pour la section 5 et au regard de la part de l'ensemble des sections $(31 \%)$, il apparait que les professeurs sont très légèrement sous-représentés dans notre discipline. Nous voyons aussi que notre taux de féminisation est inférieur à celui de l'ensemble de la profession, et que nous sommes plus "parisiens" que la moyenne. Enfin, les PR accentuent ces deux constats.

Tableau 3 : Caractérisations (mesurées en part) de la section 5 en novembre 2010.

\begin{tabular}{|l|c|c|c|c|c|c|c|}
\hline & PR & Femmes & $\begin{array}{c}\text { Femmes } \\
\text { PR }\end{array}$ & $\begin{array}{c}\text { Femmes } \\
\text { MdC }\end{array}$ & $\begin{array}{c}\text { EC dans } \\
\text { la région } \\
\text { IdF }\end{array}$ & $\begin{array}{c}\text { PR dans la } \\
\text { région IdF }\end{array}$ & $\begin{array}{c}\text { MdC dans } \\
\text { la région } \\
\text { IdF }\end{array}$ \\
\hline Section 5 & $29.5 \%$ & $32 \%$ & $17 \%$ & $38 \%$ & $28.5 \%$ & $32.7 \%$ & $23.6 \%$ \\
\hline $\begin{array}{l}\text { Ensemble des } \\
\text { sections }\end{array}$ & $31 \%$ & $36 \%$ & $22 \%$ & $42 \%$ & $24.1 \%$ & $25 \%$ & $23.7 \%$ \\
\hline Minimum & $20.6 \%$ & $13.9 \%$ & $6.2 \%$ & $18.5 \%$ & $1.98 \%$ & $1.8 \%$ & $3.3 \%$ \\
\hline Maximum & $69 \%$ & $63.8 \%$ & $50.5 \%$ & $69 \%$ & $55.6 \%$ & $55.6 \%$ & $55.2 \%$ \\
\hline
\end{tabular}

Source : GGRH A1-1 février 2011 


\section{Etude détaillée du corps des PR de la section 5 en septembre 2011}

\section{Encadré 3 : la construction du tableau de classement au 31 décembre 2011 pour les PR}

Le tableau de classement au 31 décembre 2011 constitue la liste de l'ensemble des PR et MdC par grade et ancienneté (il s'agit du fameux "cocotier"). Pour sa construction, deux bases de données mises à la disposition des membres du CNU par le MESR ont été utilisées : le tableau de classement des personnels au 31/12/2009 (le dernier cocotier connu) et le tableau des promouvables pour 2011. Ensuite, les effectifs de chaque grade ont été reconstitués de la manière suivante :

PREX2. On part du tableau de classement des personnels au 31/12/2009 et on ajoute aux effectifs du deuxième échelon de la classe exceptionnelle les promus de 2010 et de 2011 (CNU et voie locale). Comme on ne disposait pas d'informations du ministère sur les départs en retraite, des réseaux pour recenser ces collègues ont été utilisés.

PREX1. Le point de départ est le tableau des professeurs du premier échelon de la classe exceptionnelle promouvables pour 2011, auquel on rajoute les promus de 2010 et 2011 (n'ayant pas l'ancienneté requise de 18 mois dans le grade, ils ne pouvaient apparaître comme promouvables en 2011). Ensuite, on retire les promus de 2011 au deuxième échelon de la classe exceptionnelle.

PR1. Même méthodologie que pour les PR EX1.

PR2. On part du tableau des professeurs de seconde classe promouvables auquel on retranche les promus à la première classe en 2011, et on ajoute les lauréats de l'agrégation interne de 2011 et les recrutés au titre du concours 46-3.

Au delà des erreurs faites par le MESR dans la construction des tableaux des promouvables, le tableau de classement au 31 décembre 2011 comporte plusieurs autres erreurs. Il manque pour l'année 2011 à la fois les collègues recrutés au titre du 46-4, les promotions sur contingent spécifique, et les collègues en surnombre.

\section{II.1. Quelques grands traits}

Avant d'examiner à la loupe chacun des grades, nous caractérisons les PR de sciences économiques au regard de la place accordée aux femmes, de la distribution en fonction de l'âge, et de la répartition par établissement.

\section{Taux de femmes dans chacun des grades}

Le tableau 4 met en évidence une répartition inégale du taux de femmes dans chacun des grades du corps. Alors qu'un quart des professeurs de seconde classe sont des femmes, cette proportion descend à moins de $6 \%$ pour les professeurs au second échelon de la classe exceptionnelle.

Tableau 4 : Caractérisations selon le grade

\begin{tabular}{|l|c|c|c|c|c|}
\hline & PR2 & PR1 & PREX1 & PREX2 & Total \\
\hline Hommes & 124 & 175 & 84 & 65 & 448 \\
\hline Femmes & 42 & 45 & 8 & 4 & 99 \\
\hline Total & 166 & 220 & 92 & 69 & 547 \\
\hline Age moyen & 46 ans & 53 ans & 56 ans & 62 ans & 51 ans \\
& 2 mois & 3 mois & 10 mois & 9 mois & 7 mois \\
\hline Taux de femmes & $25.30 \%$ & $20.4 \%$ & $8.7 \%$ & $5.8 \%$ & $18.1 \%$ \\
\hline
\end{tabular}


Une explication de la faiblesse du nombre de femmes pour la classe exceptionnelle (12 femmes, 8 au premier échelon et 4 au deuxième échelon) pourrait être que comme le taux de féminité est en progression régulière chez les enseignants-chercheurs depuis une vingtaine d'années ${ }^{5}$, il est par conséquence plus élevé dans les tranches d'âges les plus jeunes (le tableau 4 permet de constater que l'âge moyen augmente avec le grade). Mais ceci ne suffit pas à expliquer cet effondrement du nombre de femmes chez les PREX qui n'arrivent pas à avoir accès au haut du cocotier. Une fois de plus le «plafond de verre » qui entrave leur carrière constitue une réalité indéniable. Déjà en 2007, s'appuyant sur des statistiques de 2004, un rapport du MESR sur la part des femmes constate que "ce déséquilibre s'amplifie au fur et à mesure de la formation et se poursuit au cours de la carrière professionnelle. En effet, alors que les jeunes femmes forment plus de $58 \%$ des entrants à l'université, elles ne sont plus que $46 \%$ à poursuivre en thèse et ne forment que $41 \%$ des effectifs des docteurs diplômés. Alors qu'elles représentent $36 \%$ des effectifs des chargés de recherche et de maîtres de conférences, elles ne sont plus que 18\% dans le corps des directeurs de recherche et de professeurs" [Etat des lieux de l'emploi scientifique en France, 2007]. Nous reviendrons sur ce problème dans la conclusion.

\section{Distribution du corps en fonction de l'âge}

\section{Graphique 3 : Effectifs par année de naissance}

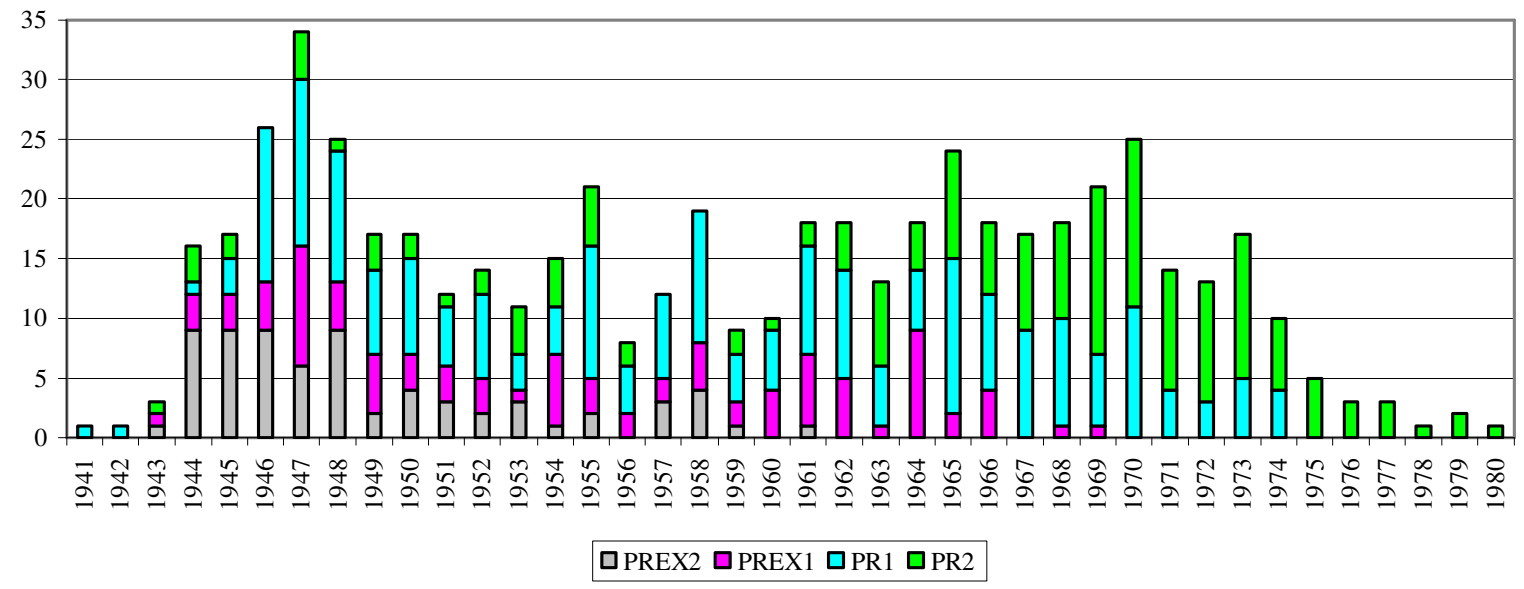

Les effectifs par année de naissance sont donnés au graphique 3. Le plus âgé est né en 1941 (70 ans), et le plus jeune en 1980 (31 ans) a été recruté en 2010 par l'agrégation externe. L'âge moyen est de 51 ans et 7 mois (soit 1 an de moins que l'âge moyen de l'ensemble des sections qui est stable depuis plusieurs années). Il est normal que la distribution des effectifs du

\footnotetext{
${ }^{5}$ Aujourd'hui $22 \%$ des professeurs sont des femmes alors que ce taux était de $14.7 \%$ il y a dix ans et de $10.4 \%$ il y a vingt ans.
} 
graphique 3 ne soit pas homogène dans les extrêmes. Du coté des plus âgés, elle dépend du choix du moment de départ à la retraite qui tend à être différé dans le temps ${ }^{6}$. Nous sommes actuellement dans un pic de départ dont le point culminant sera 2012 (fin du phénomène lié au plus "gros" du baby-boom). Pour les plus jeunes, l'entrée dans le corps des PR peu se faire à plusieurs âges en fonction des voies de recrutement (agrégation externe, interne, voie longue, voie professionnelle).

Si la répartition était uniforme sur la partie supposée homogène (avant 1948 et après 1970, qui correspondent aux âges moyens d'entrée et de sortie du corps pour l'ensemble des sections), on devrait avoir 17 PR par année de naissance. La décennie 1951-1960 est particulièrement mal représentée avec une moyenne de seulement 13 PR. Ce constat n'est pas spécifique à notre discipline et se retrouve au niveau national. Dans les extrêmes, on peut noter que 34 PR sont nés en 1947 contre seulement 8 en 1958.

\section{Répartition par établissement}

\section{Graphique 4 : Etablissements avec au moins 5 PR}

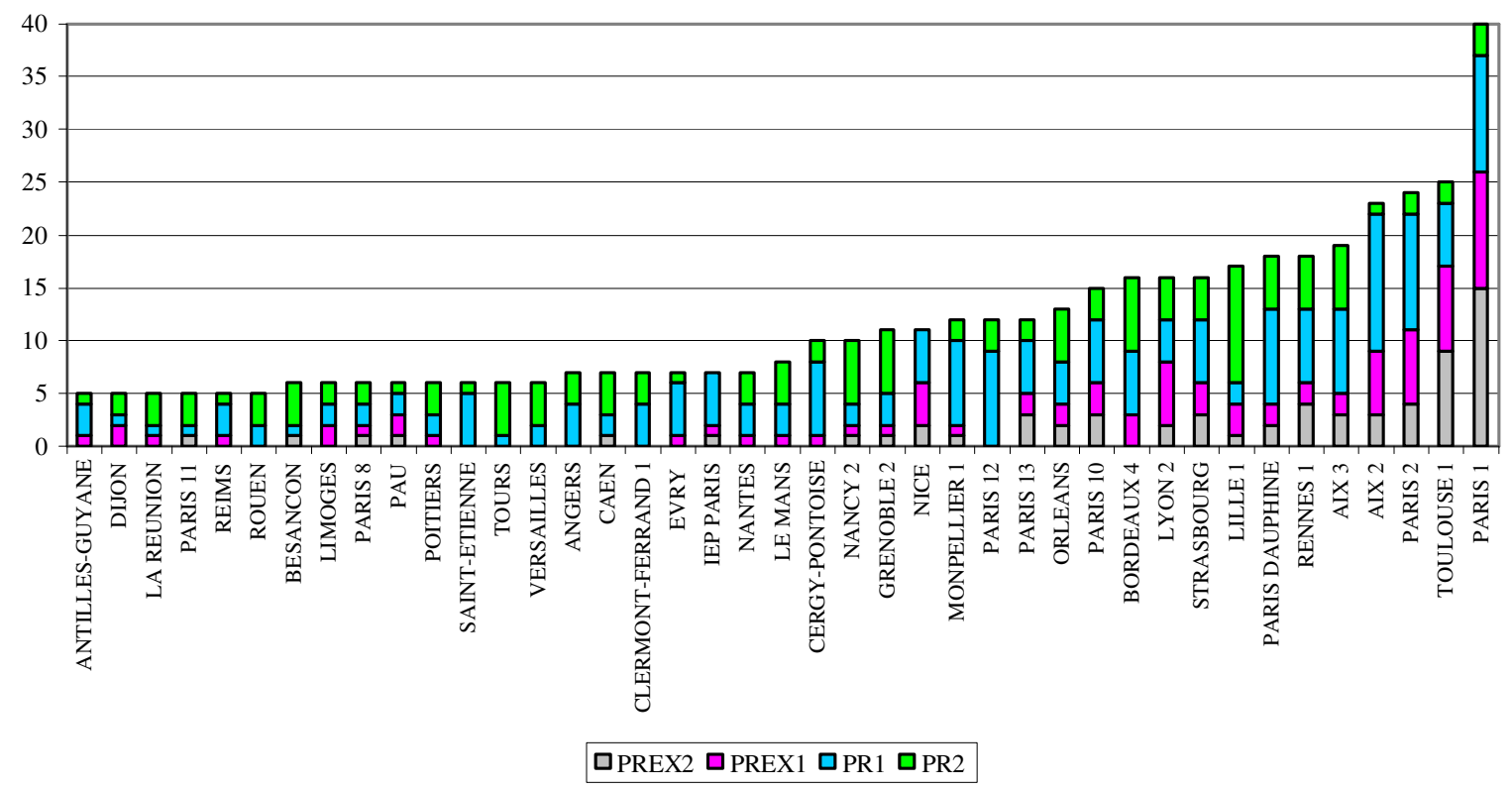

Pour l'ensemble des disciplines, la répartition géographique est d'un quart des effectifs en Ile de France et de trois quart pour les autres régions. La répartition de notre section est d'un tiers, deux tiers. Les PR sont répartis dans 83 établissements dont 5 IEP et 5 IUT. L'IEP de Paris a une place à part avec 7 PR dont aucun en deuxième classe. Les autres instituts ne comptent

\footnotetext{
${ }^{6}$ Pour l'ensemble des disciplines, en 2009-2010 les PR sont partis à la retraite à 64 ans et 6 mois et les maîtres de conférences à 64 ans et 1 mois, soit, en moyenne, dix mois plus tard qu'en 2008-2009.
} 
qu'un ou deux collègues, alors que la moyenne est à 6.6 PR par établissement et la médiane est à 4 PR. Aux extrêmes, on dénombre 21 établissements avec un seul PR, et 20 établissements avec plus de 10 PR.

Les PR2 sont affectés dans 61 établissements, les PR1 dans 65, les PREX1 dans 39 et les PREX2 dans seulement 25. L'université de Paris 1 a un effectif de 40 PR, et les établissements d'Aix-Marseille aussi (en additionnant les effectifs des trois universités).

\section{II.2. analyse des différents grades.}

Après une caractérisation rapide de chaque grade, nous nous focaliserons sur les collègues avec beaucoup d'ancienneté dans chaque grade. A partir des listes des candidats à l'avancement de grade des années quatre dernières années (2009, 2010, 2011, et 2012) nous verrons si ces collègues essayent ou non d'obtenir une promotion.

\section{L'entrée dans le corps : la deuxième classe des PR}

La deuxième classe compte 168 professeurs (42 femmes et 124 hommes), soit $30.3 \%$ du corps. Il s'agit du grade où le taux de femmes est le plus élevé, où les collègues sont les plus jeunes, et où les établissements de rattachement sont situé très majoritairement en province (139 PR2 en province contre seulement 29 en Ile de France). Parmi les universités de province, notons que les deux tiers des PR de l'université de Lille 1 sont en deuxième classe, et que sur ces 11 PR2, 6 sont des nouvelles recrues qui n'ont pas encore l'exeat. Les autres universités ayant une proportion forte de PR2 sont les universités de Tours (5 PR2 sur 6 PR), de Grenoble 2 (6 PR2 sur 11 PR), de Nancy 2 (6 PR2 sur 10 PR), et de Bordeaux 4 (7 PR 2 sur 16 PR).

\section{Graphique 5 : répartition par année d'entrée dans le grade des PR2}

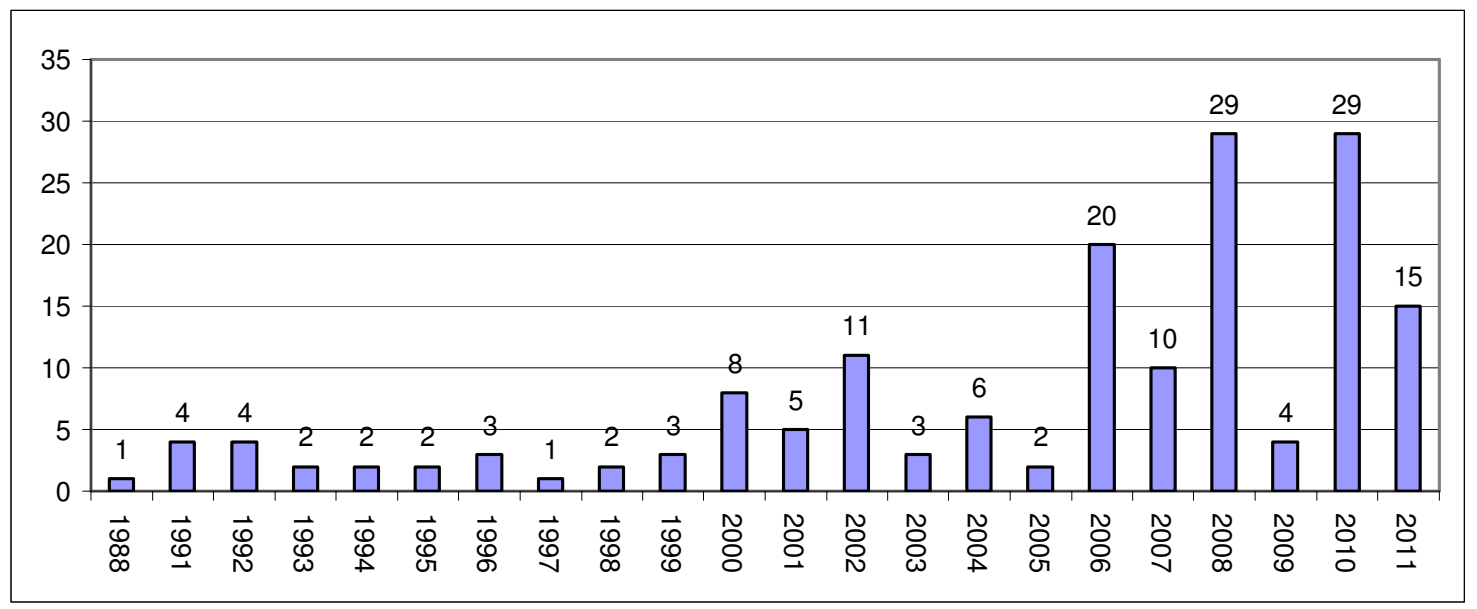


La répartition par année d'entrée dans le grade est donnée dans le graphique 5. On constate un pic des effectifs les années paires à cause de l'entrée des lauréats de l'agrégation externe (le principal mode de recrutement dans notre discipline). On voit aussi que 50\% des PR2 ont une ancienneté inférieure ou égale à 4 ans.

Il existe un consensus pour considérer que l'avancement à la première classe est une progression normale dans la carrière des professeurs des universités. Or, le graphique 5 révèle que des professeurs stagnent dans la deuxième classe, dont un depuis 23 ans. Un examen plus approfondi des collègues ayant plus de 10 ans d'ancienneté ${ }^{7}$ (entrée dans le grade depuis 2010) montre qu'ils représentent presque 20\% des effectifs. Parmi ces 34 collègues, 13 ont déposé un dossier de candidature à la promotion en 2012 (et aussi les années précédentes). Sur les 21 restant, 14 n'ont jamais déposé de dossier de candidature depuis 2009, et 7 n'ont pas déposé de dossier en 2012 alors qu'ils l'avaient fait antérieurement (candidats découragés).

\section{La première classe}

Elle est composée de 220 professeurs (45 femmes et 175 hommes), soit $40.2 \%$ du corps. Il s'agit de la classe la plus nombreuse du corps. Les collègues sont majoritairement en province, mais 37\% sont basés en région Ile de France (la part moyenne du grade étant à 32\%). Parmi les vingt universités ayant au moins 10 professeurs, on remarque que les PR1 sont majoritaires dans cinq d'entre eux : Paris 12 (9 PR1 sur 12), Cergy-Pontoise (7 PR1 sur 10), Montpellier 1 (8 PR1 sur 12), Aix-Marseille 2 (13 PR1 sur 23) et Paris Dauphine (9 PR1 sur 18). On note aussi que l'âge moyen des 13 PR1 d'Aix-Marseille 2 est de 47 ans (soit 6 ans de moins que la moyenne).

Le plus âgé est de 1941 (70 ans) et le plus jeune de 1974 (37 ans). Les âges du grade s'étalent de manière non uniforme sur 33 ans. Une fois de plus la tranche d'âge 1949-1953 est sous représentée.

Le plan en faveur des carrières dans l'enseignement supérieur et la recherche mené depuis 2009 à permis de multiplier par trois les possibilités de promotion au premier échelon de la classe exceptionnelle qui sont passés de 8 en moyenne entre 1992 et 2008 à 16 possibilités en 2009, 29 possibilité en 2010 et 24 possibilités en 2011. Cela a permis d'ouvrir un peu la promotion au premier échelon de la classe exceptionnelle qui reste cependant l'exception qui distingue une certaine excellence. Malgré cela, Il est assez difficile de sortir de ce grade et d'atteindre la classe exceptionnelle.

\footnotetext{
${ }^{7}$ Cette ancienneté permet d'atteindre le dernier échelon de la seconde classe. Il n'y a donc plus de possibilité d'avancement pour ces collègues qui risquent de subir un retard de carrière.
} 


\section{Graphique 6 : répartition par année d'entrée dans le grade des PR1}

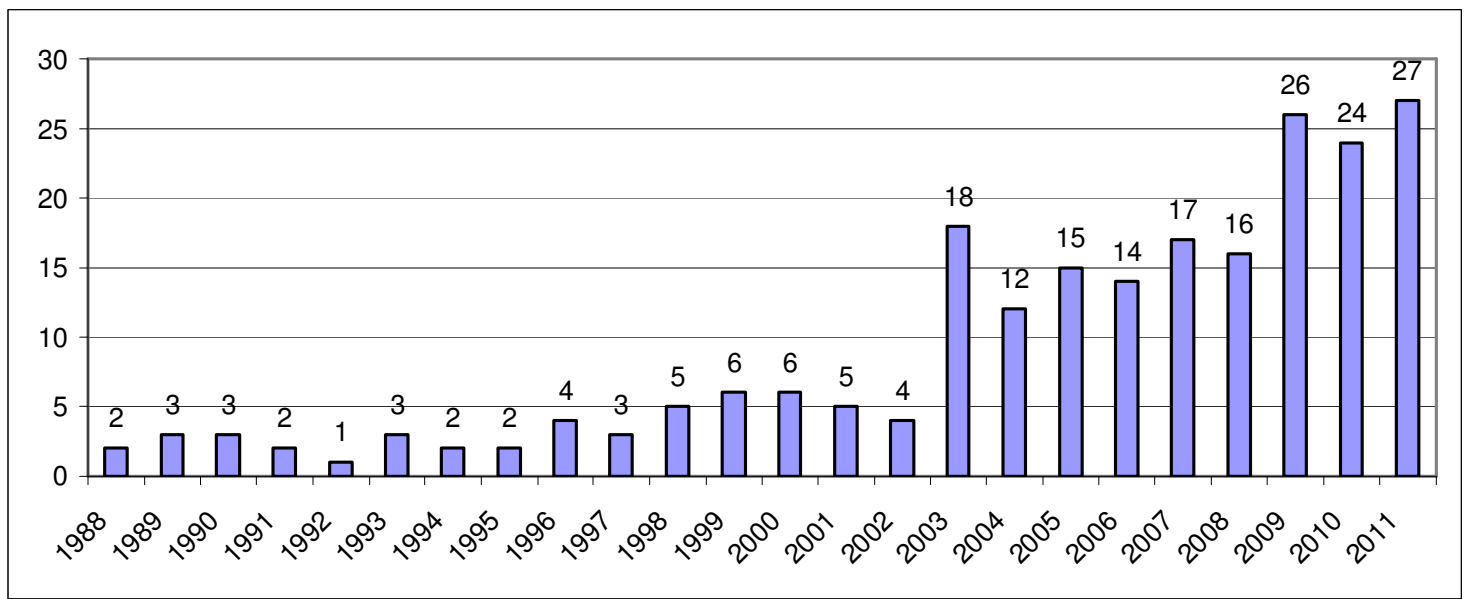

La répartition par année d'entrée dans le grade (graphique 6) laisse clairement apparaître une rupture dans les effectifs entre ceux qui sont entrés avant 2002 et ceux entrés après. La moitié des collègues ont moins de 5 ans d'ancienneté dans le grade. Le maximum d'ancienneté est de 23 ans, et 42 PR1 ont plus de 10 ans d'ancienneté (parmi lesquels 8 PR1 ont plus de 20 ans d'ancienneté).

A nouveau nous examinons en détail les 42 collègues ayant plus de 10 ans d'ancienneté dans le grade. Seuls 14 d'entre eux ont déposé un dossier de candidature à la promotion en 2012. Sur les 28 restant, 19 n'ont jamais demandé de promotion depuis 2009, et 9 ont arrêté de candidater.

\section{Un échelon de transition : le premier échelon de la classe exceptionnelle}

Il est composé de 92 professeurs (8 femmes, 84 hommes), soit 16.8\% du corps. La répartition par année d'entrée dans le grade montre qu'il s'agit d'un grade de passage, d'une antichambre soit pour la retraite ou soit pour le second échelon de la classe exceptionnelle. En effet, 85\% des membres ont au plus 3 ans d'ancienneté (la médiane est à 1 ans d'ancienneté) et l'ancienneté maximum est de 9 ans.

Une étude plus fine sur les 13 collègues ayant plus de 5 ans d'ancienneté montre qu'un seul a candidaté en 2012 (et a été promu par le CNU). Parmi les 12 restant, 7 m'ont jamais déposé de dossier de candidature depuis 2009 , et 5 se sont découragés et ont arrêté de postuler à la promotion.

La répartition par année de naissance indique que 30\% des PREX1 ont un âge inférieur ou égal à 50 ans, et qu'un quart sont proches de la retraite (au moins 63 ans). La classe d'âges 1949-1953 est sous représentée (15 personnes) alors que c'est elle qui doit monter en 
puissance et être promue au deuxième échelon de la classe exceptionnelle si on veut éviter un blocage des promotions ${ }^{8}$ dans une dizaine d'années.

L'analyse de la répartition par établissement indique une prédominance de six universités ayant une proportion de PREX1 supérieure à un quart des effectifs : Lyon 2 (38\%), Nice (36\%), Toulouse 1 (32\%), Paris 2 (29\%), Paris 1 (28\%) et Aix-Marseille $2(26 \%)$.

\section{Le haut du cocotier : le second échelon de la classe exceptionnelle}

Il compte 69 membres (65 hommes et 4 femmes), soit 12.6\% du corps. La répartition par établissement d'origine montre que Paris 1 domine avec 15 professeurs, suivi de Toulouse 1 (9), puis Rennes 1 et Paris 2 avec 4 professeurs. Les établissements parisiens totalisent 29 PREX2. Les universités de Paris 1 et l'ENS Cachan sont les établissements qui ont la proportion de PREX2 la plus élevée.

La répartition par ancienneté dans le grade indique que le plus ancien a été nommé en 1992 (20 ans d'ancienneté dans le grade !). Ceux qui ont moins de 10 ans d'ancienneté représentent $80 \%$ des effectifs et les moins de 5 ans d'ancienneté $50 \%$ des effectifs.

La répartition par année de naissance (graphique 7) est très importante car les départs à la retraite des PREX2 conditionnent les possibilités de promotions dans ce grade. 43 collègues sont nés après 1948. On peut espérer au moins une moyenne de 8 départs à la retraite chaque année durant les 5 prochaines années. Il faut faire attention dans les promotions futures à ne pas oublier la classe d'âge 1949-1956 (entre 56 ans et 63 ans) au profit de collègues plus jeunes. On risque de créer un goulot d'étranglement qui se répercutera sur les générations futures.

\section{Graphique 7 : Répartition des effectifs des PREX2 en fonction de l'année de naissance}

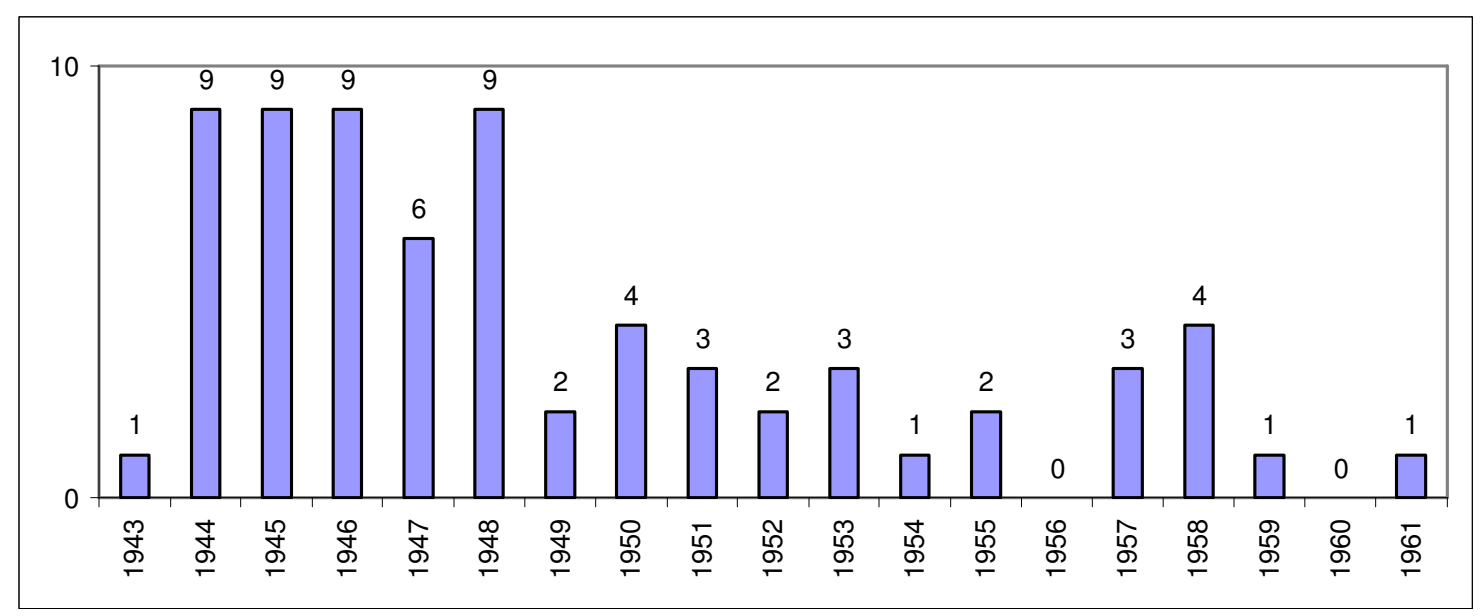

\footnotetext{
${ }^{8}$ Les possibilités d'avancement de grade au second échelon de la classe exceptionnelle ne dépendent que des départs à la retraite des membres de cet échelon.
} 


\section{Conclusion}

La conclusion de ce travail sera centrée autours de deux problèmes liés aux promotions : le premier résulte du constat une baisse inquiétante du taux de candidature (nombre de candidats divisé par le nombre de promouvables), et le second revient sur les efforts à faire concernant la promotion de nos collègues femmes.

Tableau 5 : Promotions et taux de candidature entre 2009 et 2011

\begin{tabular}{|l|l|l|l|l|}
\hline & & $\mathbf{2 0 0 9}$ & $\mathbf{2 0 1 0}$ & $\mathbf{2 0 1 1}$ \\
\hline \multirow{4}{*}{ PR2 vers PR1 } & Nombre de promouvables & 183 & 172 & 178 \\
\cline { 2 - 5 } & Nombre de candidats & 92 & 85 & 76 \\
\cline { 2 - 5 } & Nombre de promus & 24 & 24 & 27 \\
\cline { 2 - 5 } & Taux de candidature & $50.2 \%$ & $49.4 \%$ & $42.7 \%$ \\
\hline \multirow{4}{*}{ PR1 vers PREX1 } & Nombre de promouvables & 188 & 216 & 171 \\
\cline { 2 - 5 } & Nombre de candidats & 91 & 82 & 61 \\
\cline { 2 - 5 } & Nombre de promus & 16 & 29 & 24 \\
\cline { 2 - 5 } & Taux de candidature & $48.4 \%$ & $38.5 \%$ & $35.7 \%$ \\
\hline & Nombre de promouvables & 23 & 45 & 37 \\
\cline { 2 - 5 } & Nombre de candidats & 19 & 13 & 17 \\
\cline { 2 - 5 } & Nombre de promus & 6 & 6 & 10 \\
\cline { 2 - 5 } & Taux de candidature & $82.6 \%$ & $28.9 \%$ & $45.9 \%$ \\
\hline
\end{tabular}

Le premier enseignement du tableau 5 est que les possibilités de promotion augmentent depuis 2009. Cette hausse est due à l'effort de revalorisation des carrières avec l'augmentation des taux de promotion. Rappelons que sur la période 1993-2008, le nombre moyen de promus était de 19 pour le passage vers la PR1, 9 pour la PREX1, et 6 pour la PREX2 ${ }^{9}$. Le second enseignement est que le taux de candidature baisse, quel que soit le grade considéré. Nous avons un paradoxe inquiétant puisque comme le nombre de promotions augmente, la probabilité d'être promu augmente aussi, et le nombre de collègues se portant candidats à un avancement de grade devrait logiquement s'accroitre.

Pour finir, il faut insister sur l'effort que doit faire notre discipline concernant la promotion de nos collègues femmes. Une étude du MESR de 2009 a déjà pointé du doigt le problème : " (...) pour le droit, la science politique, l'économie et la gestion, la situation des femmes est sensiblement plus défavorable : elles ont été les bénéficiaires des promotions que dans $30 \%$ des cas (237 sur 790), alors qu'en moyenne sur la période, elles ont représenté environ $39 \%$

\footnotetext{
${ }^{9}$ Il est normal que le nombre de promus à la PREX2 n'augmente pas puisqu'il ne dépend que des départs à la retraite.
} 
de l'effectif du grade d'origine des promouvables ; c'est l'écart le plus grand qui est enregistré." [Etude de la promotion dans la carrière des enseignants-chercheurs 1993-2007, p 33]. Ce constat est fait sur l'ensemble des promotions MdC et PR et pour toutes les sections des groupes 1 et 2 . Il aurait été amplifié s'il n'avait porté que sur les PR de notre discipline. La même étude relève sur les 117 avancements au deuxième échelon de la classe exceptionnelle distribué entre 1993 et 2007 aux professeurs de sciences économiques, seuls 7 l'ont été pour des femmes ! Depuis un petit effort a été fait puisque sur les 22 promotions accordées dans notre disciplines pour le passage au second échelon de la classe exceptionnelle entre 2009 et 2011, 4 ont été données à des femmes ( 2 en 2009, 1 en 2010, et 1 en 2011).

\section{Bibliographie}

MESR (2012), "Campagne de recrutement et d'affectation des maîtres de conférences et des professeurs des universités Bilan de la session synchronisée 2011"

MESR (2011) "Enseignants chercheurs : répartition par discipline, région géographique (Ile de France et autres régions) et sexe", 14 février 2011.

MESR (2011) " Les personnels enseignants de l'enseignement supérieur 2009-2010", Note d'information $\mathrm{n}^{\circ} 11-06$

MESR (2010), "Etudes et regards statistiques Démographie des enseignants de l'enseignement supérieur", mars 2011

MESR (2010), "Avancement de grade des maîtres de conférences et des professeurs des universités. Bilan des promotions prononcées dans la voie spécifique d'avancement 2002 2009, avril 2010.

MESR (2009), "Etude de la promotion dans la carrière des enseignants-chercheurs 19932007".

MESR (2009) " Démographie des personnels enseignants affectés dans l'enseignement supérieur au cours de l'année 2008-2009", DGRH A1-1, juin 2009.

MESR (2009) " Les personnels enseignants de l'enseignement supérieur 2008-2009", Note d'information $n^{\circ} 09-24$

MESR (2007) "L'état des lieux de l'emploi scientifique en France", Observatoire de l'emploi scientifique, février 2007.

MESR (2006), "Etudes et regards statistiques Démographie des enseignants de l'enseignement supérieur", juin 2006

MEN (2011), "Repères et références statistiques sur les enseignements, la formation et la recherche", Editeur DEPP, Septembre 2011. 